\title{
Which Information of ICF Was Collected to Understand Our Clients?
}

\author{
Jumin Song, Haejung Lee \\ Department of Physical Therapy, College of Health \& Welfare, Silla University, Busan, Korea
}

Purpose: The purpose of the study was to review functioning components of studies for measuring functional information using International Classification of Functioning, disability and health (ICF) in Korea.

Methods: A literature review was conducted and eligible studies were identified via search of RISS, KISS, DBpia, KoreaMed, PubMed, and ISI databases published from 2001 to 2015. For inclusion in this review, a study should be published in a peer-review journal, must have participation of Korean researchers and/or developed in Korea, and should provide functioning data related ICF. Descriptive studies containing only ICF concepts and review studies were also excluded. Collected functioning data in each study were analyzed using frequency based on ICF domain. Data assessment was performed by two independent reviewers.

Results: Eighty publications were included in the analysis. The majority of studies collected data from clinical patients $(n=38)$ and from the disabled $(n=28)$. Fourteen studies assessed functioning data from the elderly and students under special education. The studies reported functioning data using various tools, including ICF itself, ICF checklist, coresets, and conventional measurement tools. Body function domain was most commonly used and the least used domain was the body structure across areas. Interestingly, increasing data related to environmental factors was observed in all studied populations.

Conclusion: Functioning data was collected from four domains of ICF across professional areas. The most common collected data was body function and activity and participation for which conventional measurement tools are already available. To understand clients, components of environmental factors that might influence a person's functioning should be considered.

Keywords: ICF, Review, Functioning

\section{서 론}

다양한 전문영역에서 치료, 사회보장 서비스, 개별화 교육 등을 개인 에게 제공하기 위해 대상자의 기능수행 관련 정보 수집이 이루어 지 고 있다.1,2 기능수행은 개인의 건강상태와 그 사람이 속해있는 개인 및 환경요인에 의해 영향을 받기 때문에 기능수행 정도를 이해하기 위해서 이들 요소에 대한 정보수집이 필요하고 이러한 정보를 바탕 으로 그 연관성과 상호관계를 분석하는 것이 중요하다.,3 또한 수집 된 정보에 대한 다양한 영역의 전문가 사이에서 의사소통이 원활할 때 대상자 중심의 서비스를 위한 다면적인 접근이 가능하다.

대상자의 기능수행 정도에 대한 수집 정보는 전문가들 사이의 의 사소통, 의사결정, 치료에 대한 법적 기록, 서비스 제공 비용 및 연구 를 위한 자료로 활용된다. 현재 임상영역에서 환자정보는 관절가동

Received Mar 22, 2016 Revised Apr 6, 2016

Accepted Apr 12, 2016

Corresponding author Haejung Lee

E-mail hjlee@silla.ac.kr
범위, 근력, 통증 등의 신체손상 중심으로 기록되고 있으며 환자의 일 상생활동작과 관련된 기능수행에 대한 정보는 매우 간략하게 서술되 어 있으며, ${ }^{5}$ 표준화된 임상측정도구인 바델 인덱스, 기능적 독립 정도 측정 도구, 요통기능수행 관련 설문지 등이 선택적으로 사용되고 있 으나 이 또한 매우 제한적으로 활용되는 실정이다. ${ }^{6}$ 장애 및 특수교육 영역에서 관련 서비스 수혜자격 결정과 개별화 교육 제공을 위해 대 상자 특성 파악은 필수적이며, 다양한 도구를 통하여 정보수집이 이 루어 지고 있다. 그러나 표준화된 다양한 측정도구를 활용하여 대상 자의 기능수행 정도를 평가하고 있으나, 기능수행에 영향을 줄 수 있 는 다양한 영역을 측정하는 데에는 제한이 있는 것으로 보고되었다.

국제 기능 장애 건강 분류(International Classification of Functioning, Disability and Health, 이하 ICF)는 2001년 세계보건기구에 의해 발 표된 국제표준분류체계 중 하나로 다양한 전문영역에서 건강 관련
Copylight (C)2016 The Korea Society of Physical Therapy

This is an Open Access article distribute under the terms of the Creative Commons Attribution Non-commercial License (Http:// creativecommons.org/license/by-nc/3.o.) which permits unrestricted non-commercial use, distribution, and reproduction in any medium, provided the original work is properly cited. 
정보의 의사소통을 위해 표준화된 도구로서 개발되었고 신체기능, 신체구조, 활동과 참여 및 환경요인 영역으로 구성되어 ${ }^{9}$ 건강과 건강 관련 개념을 다중적 관점에서 접근할 수 있는 분류체계이다. ICF는 장애가 있는 사람을 포함하여 모든 사람에게 적용할 수 있는 보편성 을 가지고 있고 ${ }^{10}$ 삶의 질 또는 환경요인의 측정, 재활과 중재효과 평 가, 교육과정 개발과 같은 연구, 임상 및 교육 도구로서 사용되고 있다.

2001년 ICF가 발표된 이후 여러 나라에서 다양한 목적으로 ICF를 활용하고 있으며, 관련 연구들의 발표도 활발하게 진행되고 있다.1 국내에서는 2004년에 ICF 한글판이 개발되었고, ${ }^{12} 2007$ 년 이후 임상, 장애 및 특수교육 영역을 포함하여 다양한 분야에서 ICF의 개념과 체계에 대한 설명, 임상적용, 기존 도구와의 연결, ICF를 활용한 데이 터수집의 분석에 대한 연구들이 발표되고 있다. 선행된 연구에서 ICF 가 특정 분야에서 어떻게 사용되며, 또한 그 활용 정도를 알 수 있고, 연구의 문제점이나 제한점 또는 향후 연구방향 등을 제시할 수 있으 므로 중요하다. 최근 선행된 ICF 관련 국내 연구들에 대한 활용 분야 와 활용 정도에 대한 현황이 보고되었다. ${ }^{13,14}$ 그러나 이들 연구에서 수 집한 기능수행 정보가 ICF 구성요소 중 어느 영역에 해당하는지 구 체적으로 분석한 연구는 미흡한 실정이다. 따라서 본 연구는 국내의 다양한 분야에서 대상자의 기능수행과 관련하여 어떤 정보를 수집 하고 있는지 그리고 수집된 정보가 ICF 구성요소 중 어느 영역에 해 당하는지를 분석하고자 한다.

\section{연구방법}

\section{1. 연구대상 논문 선정 및 자료 수집}

본 연구의 대상 자료는 RISS, KISS, NDSL, 교보스콜라, 누리미디어, KoreaMed, pubmed와 ISI database (web of science)를 활용하여 2001년 부터 2015년까지 학술지에 발표된 논문을 검색하여 수집하였다. 검 색 누락을 최소화하기 위하여 논문의 제목, 초록, 주제어에 검색어 'ICF', 'international classification of functioning, disability and health', '국 제 기능 장애 건강분류'가 포함된 논문을 검색하였다. 분석 대상논문 은 peer-review를 통해 발표된 학술논문으로 한국인 저자가 포함되고 국내에서 데이터 수집이 실시된 연구로서 ICF가 직접 활용된 원저로 하였다. 학술대회 발표논문, 학위논문, 고찰연구 및 ICF에 대한 개념 만 소개한자료는 제외하였다.

총 125 개의 논문이 검색되었다. 먼저 각 논문의 제목과 초록을 확 인하여 포함과 제외 요건을 확인하였으며, 검색된 논문에서 언급한 관련 참고문헌 또한 확인하여 검색 결과에 포함하였다. 중복 게재 논 문(1건), ICF 관련 내용이 없는 논문(4건), ICF의 개념에 대한 소개 논 문(14건), 고찰 논문(15건), ICF개념을 기반으로 개발된 도구를 사용 하였으나 직접적인 ICF항목이나 모델을 활용하지 않은 논문(9건), 일
본에서 진행된 연구논문(2건)으로 45 개의 논문을 연구분석에서 제 외하였다. 따라서 본 연구에서는 최종 80 개의 논문을 분석하였다. 논 문 검색 및 데이터 분석은 ICF 전문가 2명이 독립적으로 수행하여 결 과를 비교 확인하였다.

\section{2. 분석내용}

코딩의 준거 분석틀에 따라 연구자, 게재 연도, 연구대상자, 수집된 데이터 (평가 도구, ICF 도구 및 개인요인)를 코딩하였다. 코딩된내용 을 바탕으로 활용된 평가도구는 측정하고자 하는 내용의 개념이 연 결되는 ICF 영역으로 분석하였고, ICF 도구 또한 해당 영역으로 분류 하였다. 데이터 분석과 분류는 2 명의 전문가가 독립적으로 수행하여 그 분석 결과를 비교하였으며, 내용이 동일하지 않은 결과에 대해서 는 연구자들의 합의에 따라 최종 결정하였다. 연구대상자를 기준으 로 임상환자 대상 연구는 임상영역, 장애인 대상 논문은 장애영역, 지 역사회 노인 인구대상과 특수교육을 받고 있는 학생대상은 지역사회 영역으로 분류하였다. 각 영역에서 ICF 활용 정도를 신체기능, 신체구 조, 활동 및 참여, 환경요인 영역으로 빈도 분석하였다. 그리고 년도에 따라각 전문영역에서 활용된 ICF 영역의 변화추이도 관찰하였다.

\section{결과}

\section{1. 연구대상 영역별 분석}

본 연구의 대상인 80 건의 논문 중 연구 분야별 빈도는 임상영역( 38 건)이 가장 많았으며, 장애영역(28건), 노인과 특수교육 영역을 포함 한 지역사회영역(14건) 순으로 나타났다. 측정도구의 개념을 분석한 결과 임상영역과 지역사회영역은 신체기능이 장애영역은 신체활동 및 참여와 관련된 정보가 가장 많이 활용되는 것으로 관찰되었다. 반 면, 신체구조에 대한 정보는 전문영역에서 상관없이 가장 적게 활용 되고 있었다. ICF를 도구로서 활용한 경우, 임상과 장애영역 모두에 서 활동 및 참여에 대한 정보를 가장 많이 활용하고 있음이 관찰되었 으며, 지역사회영역의 경우는 신체기능이 다른 영역에 비해 매우 높 게 활용되고 있음을 알 수 있었다(Figure 1). 각 연구에서 활용된 측정 도구를 해당 ICF 영역으로 분석한 결과는 Table 1에 제시하였다.

\section{2. 년도별 분석}

2006년 ICF 관련 데이터 수집 논문이 발표되기 시작했고 2010년을 기 점으로 ICF와 관련된 기능수행에 대한 데이터 수집이 활발하게 발표 되었다. 임상영역에서는 환자의 기능상태에 대한 정보수집이 신체기 능과 활동 및 참여를 중심으로 이루어지고 있었으나, 환자의 기능수 행에 영향을 줄 수 있는 환경요인에 대한 정보는 2010년 이후부터 점 차적으로 증가되고 있음이 관찰되었다. 이에 반하여 장애영역에서는 


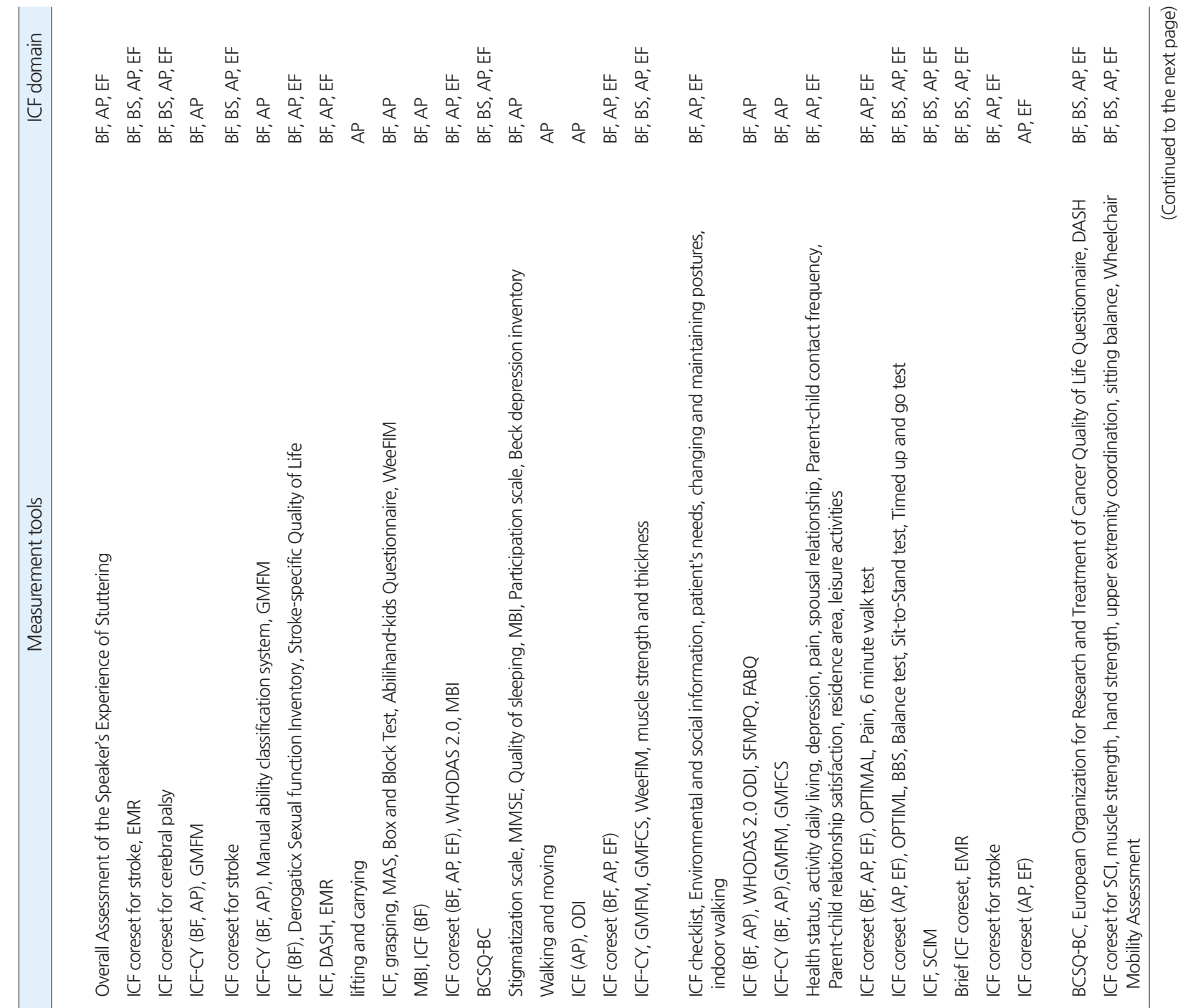

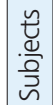
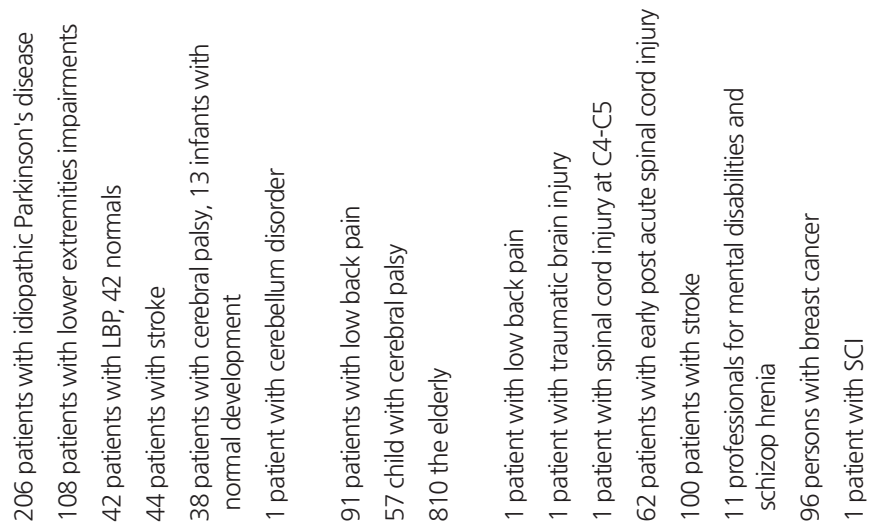

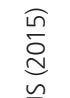

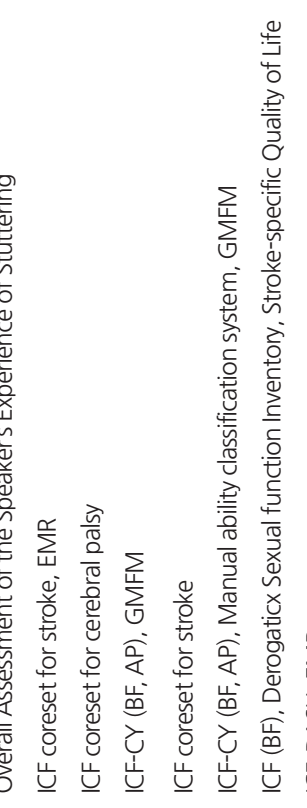

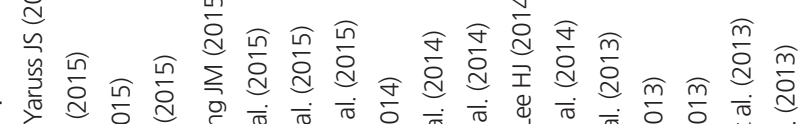

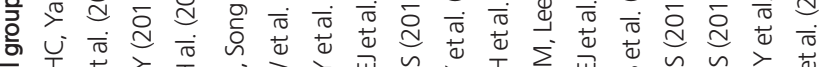

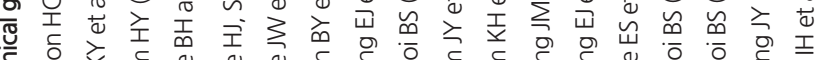

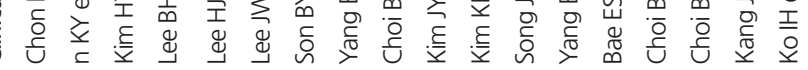




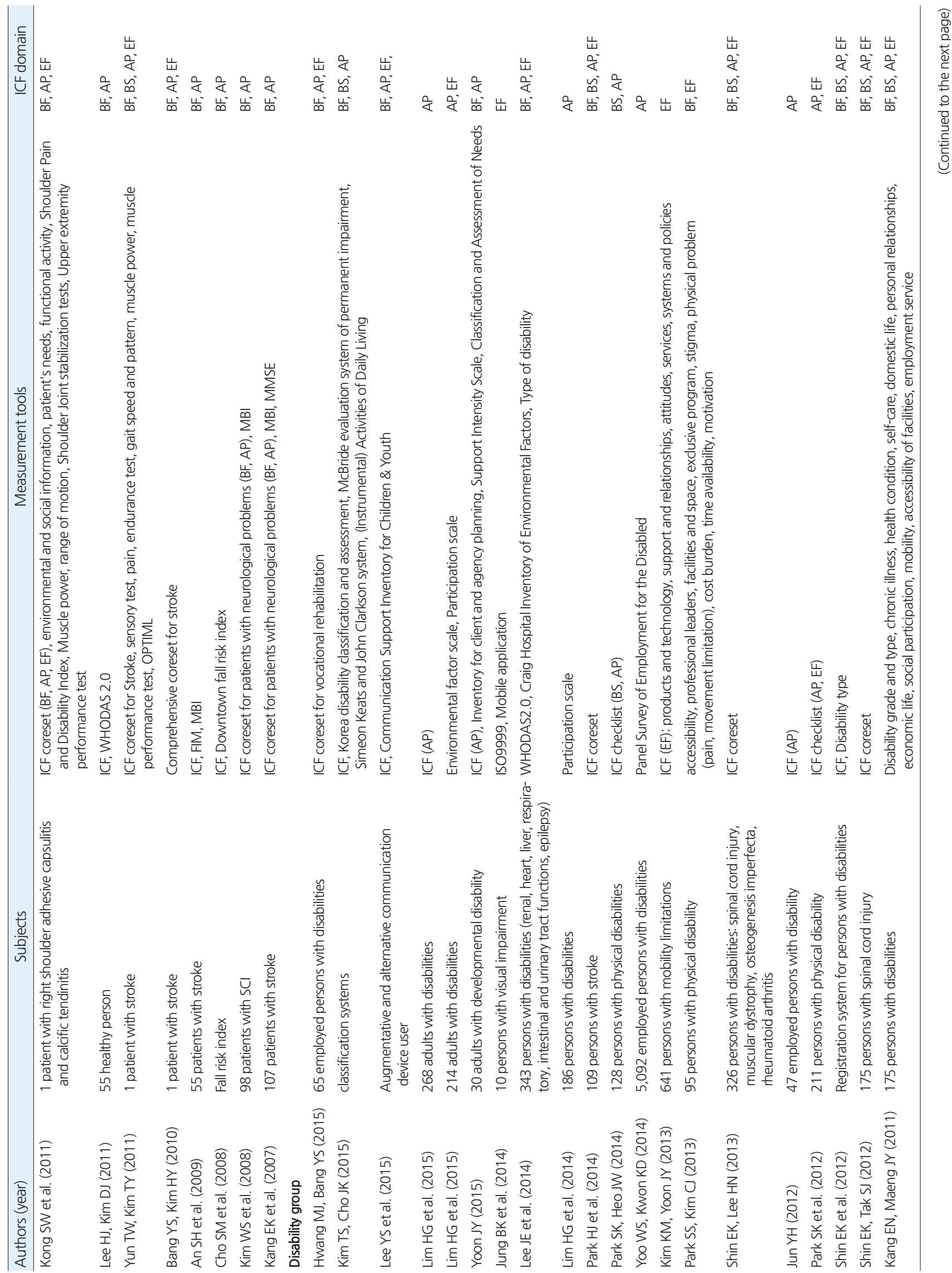




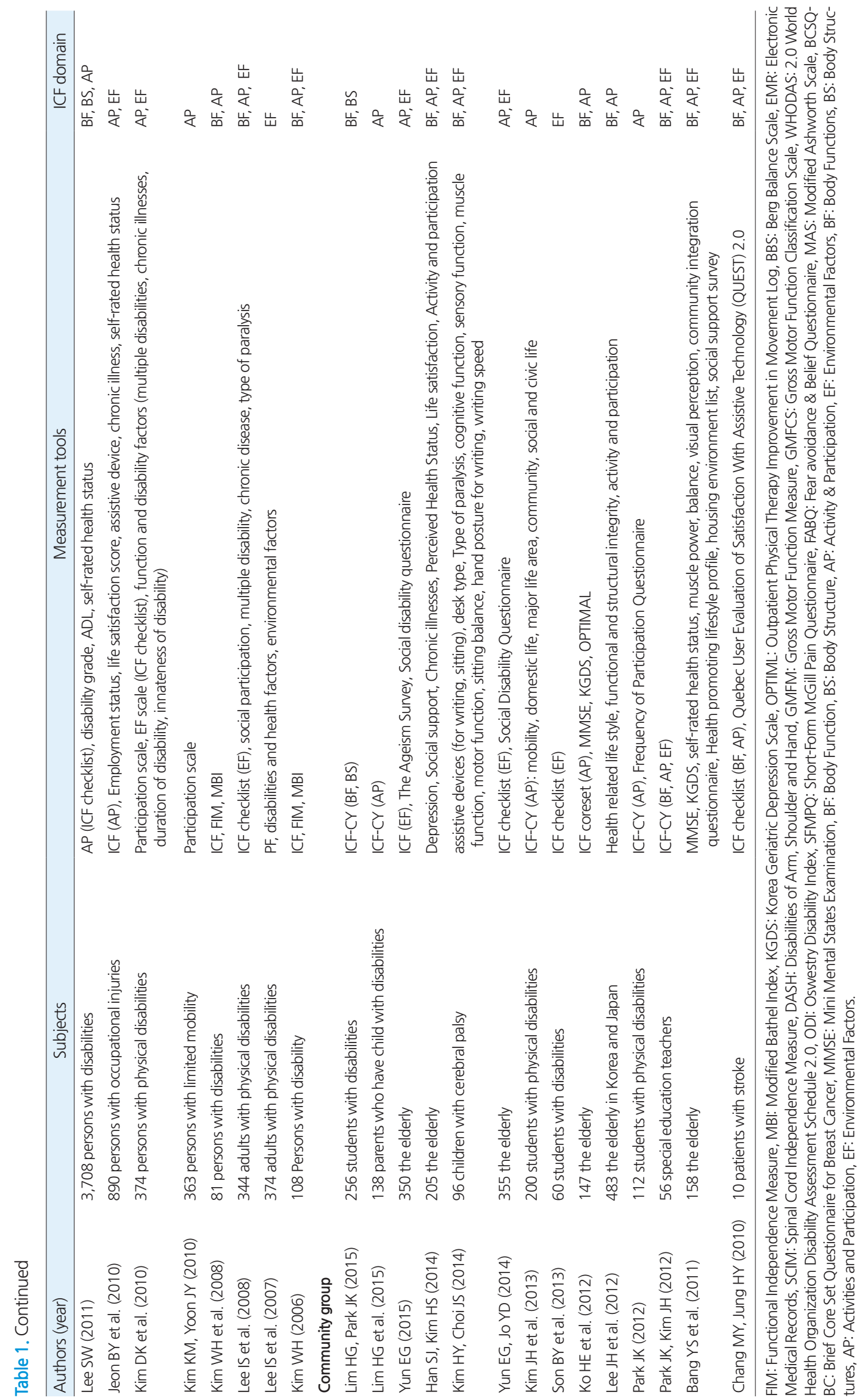



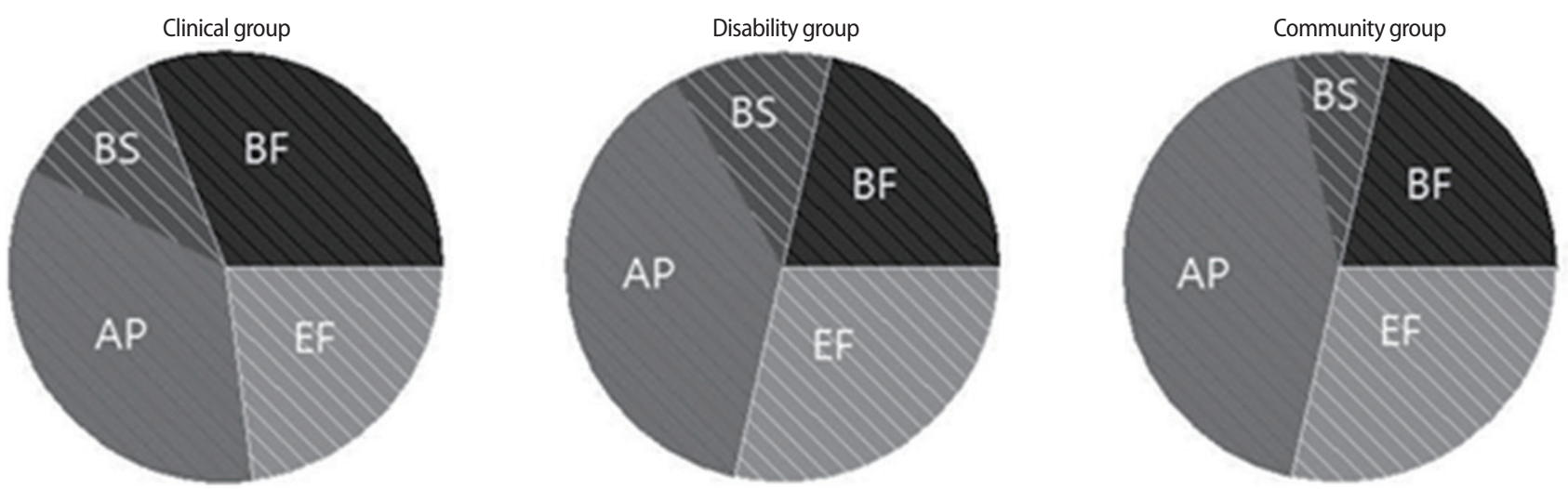

Figure 1. The proportion of measurement tools in each domain of ICF across groups: clinical, disability and community. BF: body functions, BS: body Structures, AP: activities and participation, EF: environmental factors

Clinical group

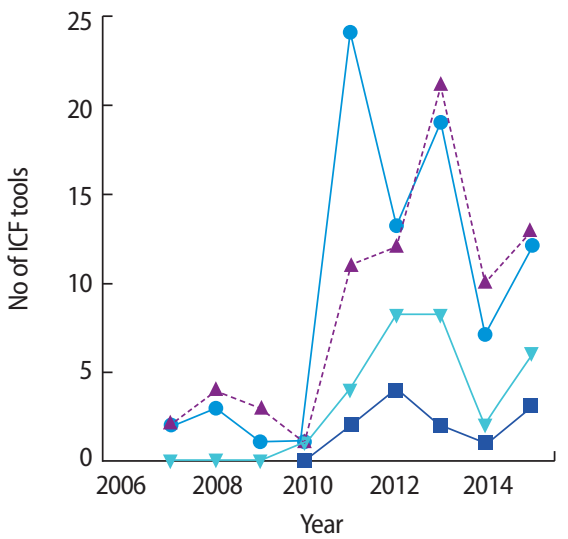

Disability group

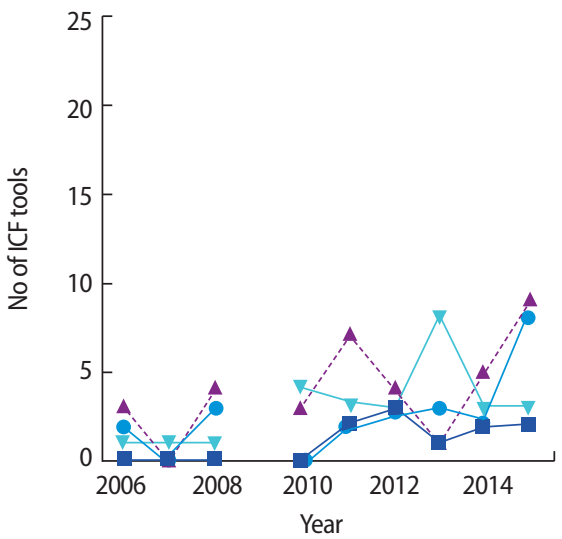

Community group

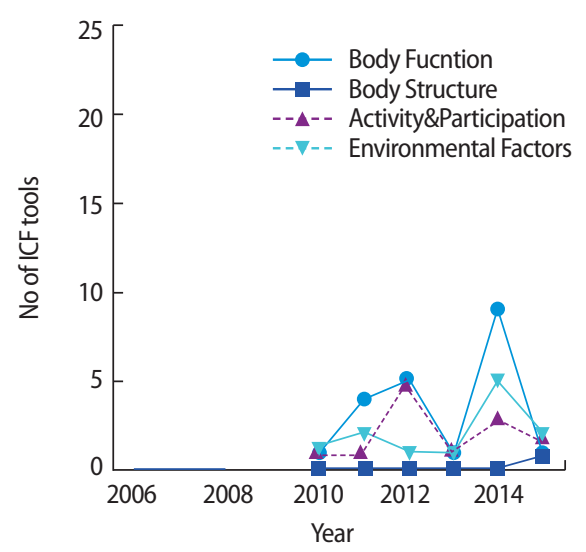

Figure 2. Date of publication and number of tools used in different domains.

환경요인에 대한 정보수집이 2006년부터 보고되고 있으며, 2010년 이 후 활발하게 수집하고 있음을 확인할 수 있었다. 지역사회영역 또한, 2010년 이후부터 신체기능을 중심으로 데이터 수집이 되고 있었고, 신체구조에 대한 정보는 전혀 활용되지 않았다(Figure 2).

\section{3. 개인요인 분석}

연령과 성별에 대한 정보는 모든 연구 영역에서 수집되었다. 임상영역 의 경우 손상부위와 건강상태에 대한 정보가 보편적으로 수집되는 반면, 장애영역에서는 직업, 경제상태, 및 가족사항에 대한 정보는 $30 \%$ 이상의 연구에서 개인정보로서 활용되었다.

\section{고 찰}

다양한 전문영역에서 치료, 사회보장서비스, 개별화 교육 등을 개인 에게 제공하기 위해 관련 정보 수집이 이루어지고 있다. 본 연구는 국 내의 임상, 장애인, 지역사회 등 다양한 영역에서 대상자의 기능수행
정도를 알기 위해 수집된 정보가 ICF구성요소 중 어느 영역에 해당 하는지를 분석하고자 총 80 편의 논문을 분석하였다.

임상영역은 2001년 ICF 가 발표된 이후 국내에서 가장 먼저 소개되 고 활용방안이 제시되었으며, 관련 연구도 가장 활발하였다..$^{3}$ 본 연 구에서도 기능수행 관련 데이터를 수집한 연구가 임상영역에서 가 장 많았다(38건). 이는 환자에게 최적의 치료를 제공하기 위해 환자의 기능수행정보 평가는 필수적이고 보건의료 전문가 사이의 의사소통 이 중요하므로 표준화된 임상 측정도구와 ICF가 많이 활용되었기 때 문으로 사료된다. 다음으로 장애인 영역에서는 장애 정도, 장애유형, 장애인 고용과 관련하여 ICF항목을 포함한 도구개발에 대한 연구와 기존 도구와 ICF를 비교하는 연구가 많았다(28건). 이러한 결과는 연 구자들이 ICF가 대상자의 상태를 이해하는 데 관련 정보수집에 표준 화된 틀을 제공하는 것으로 판단하는 것으로 생각된다.

임상영역의 연구에서 포함된 ICF 도구와 측정도구에 대한 ICF 영 역을 분석한 결과 기존 측정도구에서는 신체구조와환경요인 영역에 대한 기능수행정보가 매우 제한적이었다. 기존의 측정도구는 근력, 
균형, 보행, 정신기능 등의 신체기능에 대한 평가와 일상생활동작 수 행과 같이 평가하고자 하는 목적이 구체적이며, 이와 연관된 손상과 장애에 대한 측정도구가 매우 보편적으로 사용되고 있기 때문에 1,56 이러한 결과가 나타난 것으로 사료된다. 반면 사용된 ICF 도구에서는 ICF checklist ${ }^{15,16}$ 와 ICF coreset ${ }^{17-19}$ 가 가장 많았고, 이들 도구는 ICF의 4 개 영역을 모두 포함하고 있으므로 환경요인과 신체구조에 대한 정 보가 상대적으로 많이 수집된 것으로 판단된다(Figure 1).

장애영역의 대상논문에서 활용된 ICF 도구와 측정도구의 해당 ICF 영역은 두 가지 도구 모두에서 비슷한 패턴이 관찰되었다. 장애영 역에서는 신체기능과 활동 및 참여 정보뿐만 아니라 신체구조에 대 한 내용을 포함하는 장애등급과 유형에 대한 정보 ${ }^{20,21}$ 와 신체 기능수 행에 영향을 줄 수 있는 환경요인에 ${ }^{22,23}$ 대한 평가의 필요성을 강조하 기 때문으로 생각된다(Figure 1).

지역사회영역의 기존 측정도구의 해당 ICF 영역에서 신체구조에 대한 정보는 관찰되지 않았으며 이러한 결과는 특수교육이나 지역사 회에서의 노인활동과 관련된 기능수행 정보에는 신체구조에 대한 내 용이 직접적으로 활용되지 않는 것으로 판단된다. 반면 신체기능에 대한 정보가 상대적으로 많이 수집되는 것으로 관찰되었고 지역사 회활동에 신체기능 손상 정도가 활동과 참여에 영향을 준다는 전통 적인 사고가 바탕이 되어있는 것으로 사료된다. ${ }^{2426}$ 본 연구에 포함된 지역사회영역 대상논문의 수가 매우 제한되어 단정지어 결론을 도출 하기에는 어려움이 있다(Figure 1).

수집한 데이터를 연도에 따라 분석한 결과 2004년 ICF 한글판이 보급된 이후 2006년부터 꾸준히 ICF를 직접적으로 활용한 데이터 수 집 논문이 발표되기 시작했으며, 2010년을 기점으로 더욱 활발하게 연구됨이 관찰되었다. 임상영역에서는 신체기능과 활동 및 참여에 대한 정보 수집이 지속되었고 2011년 이후 환경요인에 대한 정보도 활용되고 있음을 알 수 있었다. 이는 환자 중심의 치료를 위해 환자가 속한 환경에 대한 정보를 수집하여 기능수행을 증진하기 위한 촉진 요인으로 적용하고자 하는 노력의 일환으로 사료된다.27 장애영역에 서는 환경요인에 대한 정보수집이 2006년부터 관찰되었으며, 이는 장 애영역에서는 대상자들의 생활에서 환경요인에 대한 중요성을 인식 하고 있기 때문으로 생각된다. ${ }^{23}$ 지역사회영역에서는 발표된 연구가 2010년 이후부터 신체기능과 환경요인을 중심으로 데이터 수집이 이 루어지고 있어 다른 전문 영역보다 ICF 적용이 다소 늦은 것이 관찰 하였다.

장애영역에서는 직업, 경제상태, 학력, 가족사항 등 개인요인에 대 한 정보가 다른 전문영역에 비하여 매우 다양하게 수집되었다. 이는 배경요인 중 개인요인이 개인의 기능수행과 밀접한 관계가 있다는 것 을 보여 준다.28-30

각 연구에서 활용된 측정도구를 해당 ICF 영역으로 분석한 결과
는 신체 기능 및 활동과 참여영역에 해당하는 측정도구가 가장 많이 활용되었다. 수정된 Ashworth 척도, 버그균형척도, Beck 우울척도, 간 이정신상태검사, 맥길통증척도는 신체손상과 관련된 항목으로 구성 되고, 6 분걷기와 같은 보행검사, 수정바델인덱스는 활동에 대한 항목 으로 구성되어 ICF의 단일 영역에 해당될 수 있다. 반면, 기능적 독립 평가, 상지기능장애척도, 요통기능척도, WHODAS 2.0 의 항목들은 $\mathrm{ICF}$ 두 개의 영역, 즉 신체기능과 활동 및 참여영역으로 분석될 수 있 다. 이와 같은 기존 측정도구의 항목들은 진단과 같은 특정목적을 위 해 구체적인 항목으로 구성되어 있으나, 개인의 전반적인 기능수행에 영향을 줄 수 있는 환경요인과 같은 다양한 요소를 확인하기에는 제 한적이다. 대상자에게 적절한 서비스를 제공하기 위해서는 관련 측 정도구 선택이 중요하다. 개인이 가정, 직장, 지역사회, 학교에서의 역 할 수행은 개인이 속한 주변 환경을 비롯하여 다양한 측면에 의해 영 향을 받는다. 그러므로 대상자 중심의 접근을 위해 필요한 서비스 내 용에 적합한 측정도구가 선택되어야 한다. 임상, 장애, 특수교육을 포 함한 지역사회영역에서 ICF의 모든 영역과 관련된 정보가 수집되고 있음을 알 수 있었다. 기존의 측정도구에 신체기능과 활동 및 참여에 대한 항목이 포함되어 있어 수집된 정보 역시 이와 관련된 내용이 많 았다. 그러나 대상자의 기능수행 정도를 잘 이해하기 위해서는 기능 수행 정도에 영향을 줄 수 있는 환경요인에 대한 충분한 정보가 필요 하다. 본 연구에서는 각 기능수행정보를 ICF 영역별로 분석하여 제시 하였으나, 대상자의 기능수행 정도를 이해하기 위해 측정도구 항목 을 포함하여 수집된 모든 관련 정보에 대한 항목별 평가가 필요하며, 또한 ICF 항목의 분류척도에 대한 연구가 필요하다고 생각된다.

\section{REFERENCES}

1. Lee H, Song J. Analysis of the characteristics of patients with chronic low back pain using the ICF concept. J Kor Phys Ther. 2013;25(5):282-7.

2. Park JK, Kim JH. Basic research of utilizing ICF-CY for the development of the IEP for students with disabilities. Kor Council Phys Multi \& Health Disabil. 2012;55(3):47-70.

3. Lee SE. Factors affecting leisure participation of older adults with arthritis. J Leisure Studies. 2013;11(1):25-41.

4. Son BY, Bang YS, Kim HY et al. ICF environmental factors related to university life adjustment of students with disabilities. Kor Entertainment Industr Assoc. 2013;7(2):71-8.

5. Lee H, Song J. Which information is commonly used for patients with stroke at rehabilitation settings? J Kor Phys Ther. 2015;27(6):392-99.

6. Song J, Lee H. Is the sis 3.0 valid for use at a rehabilitation setting in korea for patients with stroke? J Kor Phys Ther. 2015;27(4):252-57.

7. Shin HU, Kang B. A study on development of the job functional assessment scale for people with disabilities. J digit convergence. 2014;12(4): 379-97.

8. Shin EK, Shin HI, Lee HN. A study on the application of relevant ICF 2 
level codes in Korea. J Soc Welfare Rev. 2012;32(4):490-529.

9. WHO. International classification of functioning, disability and health. 2001.

10. WHO. How to use the ICF: a practical manual for using the international classification of functioning, disability and health (ICF). Exporsure draft for comment. Geneva, WHO, 2013.

11. Stucki G. International classification of functioning, disability, and health (ICF): a promising framework and classification for rehabilitation medicine. Am J Phys Med Rehabil. 2005;84(10):733-40.

12. Ministry of Health \& Welfare. International classification of functioning, disability and health-Korean version. WHO, 2004.

13. Lee H, Song J. An overview of the ICF's use in Korea. J Kor Phys Ther. 2015;27(5):356-63.

14. Kim SW, Kim JY. Analysis of domestic research trends related to international classification of functioning, disability and health (ICF). J Special Edu \& Rehabil Sci. 2015;54(3):149-72.

15. Kim DK, Kim SY, Lee SJ. Predictors of social participation of people with severe physical disabilities based on ICF framework Humanities \& Soc Sci Res. 2010;28:5-39.

16. Lee I, Kim S, Choi J. A exploratory study on factors affecting disability identity of people with physical disabilities. J Rehab Res. 2007;11(3):5676.

17. Yang EJ, Kim BR, Shin HI et al. Use of the international classification of functioning, disability and health as a functional assessment tool for breast cancer survivors. J Breast Cancer. 2012;15(1):43-50.

18. Nam HS, Kim KD, Shin HI. ICF based comprehensive evaluation for post-acute spinal cord injury. Ann Rehabil Med. 2012;36(6):804-14.

19. Song J, Lee H. Investigating functional level in patients with stroke using ICF concept. J Kor Phys Ther. 2014;26(5):351-7.

20. Park S, Heo J. Correlation between structures related to movement, activity and participation in disabled persons living at home. J Kor Phys Ther. 2014;26(2):130-5.

21. Lee SW. Effects of work ability on the employment status of people with disabilities: comparing degrees of disability, ICF activity limitations \& participation restriction, levels of assistance needed for daily activities, and self-assessed health status. Kor J Soc Welfare Studies. 2011;42(2): 323-50.

22. Kim WH, Ahn MR, Kim ES et al. Function and environmental factors analysis using ICF (inernational classification of functioning, disability and health) for people with disabilities. J Kor Academy Rehab Med. 2008;32(1):100-5.

23. Kim KM, Yoon JY. Development of a scale to measure environmental factors Disabil \& Employment. 2013;23(1):189-208.

24. Kim HY, Choi JS. An investigation of the factors affecting handwriting speed on school aged children with cerebral palsy based on international classification of functioning, disability and health. J Spec Edu \& Rehab Sci. 2014;53(4):53-69.

25. Ko HE, Oh MH, Baek JY et al. A comparative study of communitydwelling elderly people body functions and activities \& participations based on ICF. J Kor Soc Occup Ther. 2012;20(4):15-28.

26. Bang YS, Ryu SY, Kim HY. The relationship between the components of ICF and self-rated health status in the elderly who use senior citizen center. J Regional Studies. 2011;19(4):95-113.

27. Ministry of Health \& Welfare. International classification of functioning, disability and health-Korean version. 2nd Ed. WHO, 2016.

28. Park HJ, Bang YS, Kim HY. Factors relating community living participation in elderly people with stroke using international classification of functioning, disability and health. Kor Entertainment Industr Assoc. 2014;8(2):215-25.

29. Lim HG, Park JK, Kim ER. Actual condition and recognition on social participation of individuals with disabilities. J Spec Child Edu. 2014; 16(2):519-43.

30. Shin EK, Tak SJ. Study on affecting factors on work and employment of the persons with spinal cord injury: the application of ICF coresets. J Rehab Res. 2012;16(3):133-63. 